\title{
Bayesian multilevel model on maternal mortality in Ethiopia
}

\author{
Shibiru Jabessa ${ }^{1 *}$ (1) and Dabala Jabessa ${ }^{2}$
}

\author{
${ }^{*}$ Correspondence: \\ sjabessa@yahoo.com \\ 1 Department of Statistics, \\ College of Natural \\ and Computational Sciences, \\ Wollega University, Nekemte, \\ Ethiopia \\ Full list of author information \\ is available at the end of the \\ article
}

\begin{abstract}
Maternal mortality is one of the socio-economic problems and widely considered a serious indicator of the quality of a health. Ethiopia is considered to be one of the top six sub-Saharan countries with severe maternal mortality. The objective of this study was to investigate the effects of the Demographic and Socio-economic determinant factors of maternal mortality in Ethiopia. Data from the 2016 Ethiopia Demographic and Health Survey indicated that the sample of women (15-49) was $(n=10,103)$. The Bayesian multilevel we were used to explore the major risk factors and regional variations in maternal mortality in Ethiopia. Markov chain Monte Carlo methods with non-informative priors have been applied. The Deviance Information Criterion model selection criteria were used to select the appropriate model. The analysis result, 145 (1.43\%) mothers were died due to pregnancy. Using model selection criteria Bayesian multilevel random coefficient was found to be appropriate. With this model, Age of mother, marital status, number of living children, wealth index and Education are found to be the significant determinants of maternal mortality in Ethiopia. The study indicated that there was within and between regional variations in maternal mortality. Inference is the fully Bayesian multilevel model based on recent Markov chain Monte Carlo techniques. The socioeconomic, demographic and environmental determinants included in the study were found to be statistically significant. The result of the Bayesian multilevel model in this study has shown that educational attainment, wealth index, an age of mother, status and number of living children was a significant factor of maternal mortality.
\end{abstract}

Keywords: Demographic and Health Survey 2016, Ethiopia, Bayesian multilevel, Random Intercept Logistic Regression Model, Maternal mortality

\section{Introduction}

\section{Background of the study}

Maternal mortality is known as maternal death and major cause of death among women of reproductive age [48]. As explained in Shah and Say [40], maternal mortality is the death of a woman while pregnant or within 42 days of termination of pregnancy. It caused by any related of the pregnancy but not from accidental or incidental causes. As World Health Organization, 2012 indicates that annually, 287,000 women die globally during a complication of pregnancy and child birth. The overwhelming majority of 
maternal deaths occurs in low resource countries and arises from the risks attributable to pregnancy and childbirth as well as from the poor performance of health services [15].

Developing countries accounted for $99 \%(533,000)$ of the deaths. Slightly more than half of the maternal deaths $(270,000)$ occurred in the sub-Saharan Africa region alone, followed by South Asia 188,000 [20]. At the country level, India at 19\% $(56,000)$ and Nigeria at $14 \%(40,000)$ account for a third of global maternal deaths. In sub-Saharan Africa, one out of every 13 women dies of pregnancy-related causes during their lifetime as with one in 4,085 women in industrialized countries [28].

Ethiopia has one of the highest rates of maternal mortality in sub-Saharan African countries [49]. Considering the goal of reducing maternal mortality and improving maternal health problem, it is essential to understand the factors that affect maternal mortality in order to find the possible solutions for improving maternal health problem in Ethiopia [12].

As WHO, 2015 report Ethiopia is one of top six countries that contribute to about $50 \%$ of maternal mortality [5]. Demographic and Health Survey data is hierarchical structure, in such case two sources of random variation was available. Thus the study was adopted Bayesian multilevel models, to examine the impact of contextual factors on maternal mortality and its variations among regions. However, most of those studies were based on logistic which cannot be empowered to answer there were geographical variations or not $[34,47]$. Besides, the other studies were conducted at hospital level with limited covariates; and based on the classical models that have relative drawback [2, 17]. We suggested that there was a variation within and between the community levels [29]. Hence, this study was intended to entire the gap by considering the random effects under multilevel model of Bayesian paradigm [1,25].

With the efforts so far made by the government to bring MMR down, it is still not yielding the desired result which was developed by the study developed on the trends in maternal mortality between 1990 and 2015 years [46]. It is therefore of reasonable interest to identify and explain the existence variations due to the random effects at women and regional levels and subsequently, to determine the effects of the Demographic and Socio-economic determinant factors on maternal mortality in Ethiopia (Fig. 1).

Estimates from EDHS surveys indicate a substantial decline in the pregnancy-related mortality ratio in Ethiopia since 2000, from 871 deaths per 100,000 live births in the 7 years before the 2000 EDHS survey to 673 deaths per 100,000 live births in the 7 years before the 2005 EDHS survey, 676 deaths per 100,000 live births in the 7 years before the 2011 EDHS survey, and 412 deaths per 100,000 live births in the 7 years before the 2016 EDHS

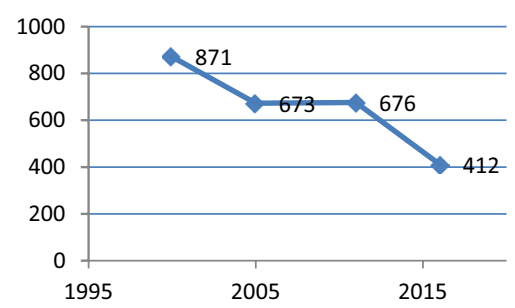

Fig. 1 Trends of Maternal mortality rates per 100,000 
survey [13]. Despite the decrease in maternal deaths over the years, the number is still highest among the world and unacceptably high [23].

According to different previous study, the advantage of Bayesian approach over that of classical method has been certified. However, most of those studies were based on logistic which cannot be empowered to answer there were geographical variations or not [34, 47]. Besides, the other studies were conducted at hospital level with limited covariates, and based on the classical models that have relative drawback [2, 17]. Hence, this study was intended to entire the gap by considering the random effects under multilevel model of Bayesian paradigm [33]. The basic research questions are: which variables have significant impacts on maternal mortality from the study variables, is there variation of maternal mortality within and between Regional States of Ethiopia and from the study variables which predictors have variation across regions?

The general objective of this study is to identify and explain the effects of the demographic and socio-economic determinant factors on maternal mortality in Ethiopia. And, to identify the factors associated with maternal mortality in Ethiopia, to examine the extent of the variation within and between regional variations of maternal mortality in Ethiopia and to determine from the study variables, the variation of predictors across regions.

\section{The significance of the study}

Despite the amount of work published as well as policies and initiatives being adopted in an effort to reduce maternal death; the problem are still not clear. Previous study was identify some variable for the cause and risk at specific level like hospital or district [51]. However these only present the level of maternal mortality at individual level not at national level [22]. This research fills the statistical analysis by adopting an appropriate method which is hierarchical by its nature [8]. In general; this research has a significant role for our country to identify the most serious determinants of maternal mortality. Bayesian multilevel logistic regression model that will help to take action on those identified determinants. Finally, this study would stimulate further research in the application of Bayesian multilevel model in the area of maternal health and mortality.

\section{Methodology}

Source of the data: The data used in this study is EDHS, 2016 which was implemented by the CSA and $\mathrm{MoH}$. A total of sample of 10,103 women between the ages of 15-49 years in Ethiopia was included in this study. All women age 15-49 and all men age 15-59 who were either permanent residents of the selected households or visitors who stayed in the household the night before the survey were eligible to be interviewed [13].

Variable of the study: In this study, the potential determinant factors expected to be correlated with pregnancy-related death are included as variables.

Response variable: The response variable in this study is the survival status of mothers at a reproductive age and this variable is dichotomous, coded as 1 if death due to pregnancy has occurred and 0 otherwise.

$$
Y_{i j}= \begin{cases}1 \text { if the ith women are dying in the } j \text { th region } \\ 0 & \text { otherwise }\end{cases}
$$


The predictor variables: Many explanatory variables were used as predictors of maternal mortality. The explanatory variables that included in this study were:

Place of delivery

Antenatal care

Mother's age at birth

Place of residence

Region Mothers education

Marital status

Wealth index

Contraceptive

Number of living children

Source of drinking water

\section{Method of data analysis}

The statistical model that used for this data to analysis was the Bayesian multilevel logistic model. The Markov chain Monte-Carlo (MCMC) method is a general simulation method for sampling from posterior distributions and computing posterior quantities of interest [18]. Sampling process of MCMC approaches is pretty heavy but has no bias and, so, these methods are preferred when accurate results are expected, without regards to the time it takes [24]. The association between water pipe dependence and chronic obstructive pulmonary disease, by comparing frequentist and Bayesian methods' results show as Bayesian approach have advantages over the frequentist one, particularly in case of a low power of the frequents analysis [38].

The data collection procedure is the hierarchical level or structures that means the levels are nested one another; Kinds of the literature indicated that the Bayesian models are given preference because the technique is more robust and precise than the traditional (classical) statistics since it is usually criticized based on the priors and information from the likelihood. Thus, this collective information has been strengthening the better determination of the parameter [34, 35]. Bayesian multilevel logistic regression model has been helped to facilitate the representation of complex multilevel data structures, the variation among the women and the region the specification of objective priors and also gives us more informative and quantitative answers than any of the standard frequents approaches [39], thus why the reason for selecting this model. MLwiN 2.02 version software was adopted for the analysis of this study.

Multilevel Logistic Regression Model: Multilevel hierarchical modeling explicitly accounts for the clustering of the units of analysis, individuals nested within groups. The study helps for examination of the effects of group level and individual level variation- of observations. We further simplify the presentation by assuming there is a women-level predictor and regional level factor of maternal mortality. Multilevel models are statistical models which allow not only independent variable at any level of a hierarchical structure but also at least one random effect above level one group. A multilevel logistic regression model can account for lack of independence across levels of nested data (i.e., individuals nested within regions). For simplicity of presentation two-level models for this study, i.e., models accounting for women-level and regional -level effects. In this data structure, 
level-1 is the women level and level-2 is the regional level. Within each level- 2 unit, there is $n_{j}$ in the $j$ th region.

The standard assumption is that $\mathrm{Y}_{\mathrm{ij}}$ has a Bernoulli distribution [10]. Then, the twolevel models are given by:

$$
\begin{aligned}
& \log i t\left(\pi_{i j}\right)=\log \left[\frac{\pi_{i j}}{1-\pi_{i j}}\right]=\beta_{0 j}+\sum_{h=1}^{k} \beta_{h j} x_{i j k} \\
& i=1,2,3, n_{j}, h=1,2, \ldots k, j=1,2, \ldots, 11 \\
& \beta_{0 j}=\beta_{0}+u_{0 j} \\
& \beta_{1 j}=\beta_{1}+u_{1 j}, \ldots, \beta_{k j}=\beta_{k}+u_{k j} \\
& \log i t\left(\pi_{i j}\right)=\log \left[\frac{\pi_{i j}}{1-\pi_{i j}}\right]=\beta_{0}+\sum_{h=1}^{k} \beta_{h j} x_{i j k}
\end{aligned}
$$

$x_{i}=\left(x_{1 i j}, x_{2 i j}, \ldots, x_{k i j}\right)$, represent the first and the second level covariates, for variable $\mathrm{k}$. $\beta\left(=\beta_{0}, \beta_{1}, \ldots, \beta_{k}\right)$, are the regression parameter coefficient.

$\left(u_{0 j}, u_{1 j}, \ldots, u_{k j}\right)$, is the random effect of the model parameter at level two. With the assumption, $u_{h j}$ follows a normal distribution with mean zero and variance $\sigma_{u}^{2}$ without $\mathrm{U}_{\mathrm{hj}} u_{h j}$ the above equation can be the single-level logistic regression. That means the $1^{\text {st }}$ equation is the single level logistic model and the 2 nd equation is two levels model. Therefore conditional on $u_{0 j}, u_{1 j}, \ldots, u_{k j}$ the $y_{\mathrm{ij}}$ can be assumed to be independently distributed as Bernoulli random variables.

$x_{i}=\left(x_{1 i j}, x_{2 i j}, \ldots, x_{k i j}\right)$, represent the first and the second level covariates, for variable $\mathrm{k}$. $\left(\beta=\beta_{0}, \beta_{1}, \ldots, \beta_{k}\right)$, are the regression parameter coefficient. $u_{0 j}, u_{1 j}, \ldots, u_{k j}$, is the random effect of the model parameter at level two. With the assumption, $u_{h j}$ follows a normal distribution with mean zero and variance $\sigma_{u}^{2}$.

\section{Bayesian multilevel analysis of empty model (null model)}

It is the probability distribution for group-dependent probabilities without taking further explanatory variables into account.

The null model is defined as:

$$
\operatorname{logit}\left(\pi_{i j}\right)=\beta_{0}+U_{0 j}
$$

The index $\mathrm{i}$ indicates individual for level one, $\mathrm{j}$ indicates region for level two, $U_{0 j}$ is level two errors, $\beta_{0}$ is the overall average of maternal mortality.

\section{Bayesian multilevel analysis of random intercept model}

In the random intercept model the intercept is the only random effect meaning that the groups (region) differ with respect to the average value of the response variable.

The random intercept model expresses the logit of $\pi_{i j}$ is a sum of the linear function of explanatory variables and given as: 


$$
\operatorname{logit}\left(\pi_{i j}\right)=\log \left[\frac{\pi_{i j}}{1-\pi_{i j}}\right]=\beta_{0 j}+\beta_{1} X_{1 i j}+\cdots+\beta_{k} X_{k i j}=\beta_{0 j}+\sum_{h=1}^{k} \beta_{h i j}
$$

where the intercept term $\beta_{0 j}$ is assumed to vary randomly and is given by the sum of an average intercept $\beta_{0}$ and group-dependent deviations $U_{0 j}$ that is $\beta_{0 j}=\beta_{0}+U_{0 j}$ as a result.

$$
\operatorname{logit}\left(\pi_{i j}\right)=\beta_{0}+\sum_{h=1}^{k} \beta_{h j} x_{h i j}+U_{0 j}
$$

where $\beta_{0}+\sum_{h=1}^{k} \beta_{h j} x_{h i j}$ is the fixed part of the model and $U_{0 j}$ is the random or stochastic part of the model.

\section{Bayesian multilevel analysis of random coefficients model}

The multilevel random effect coefficients logistic regression model is based on linear models for the log odds that include random effects for groups or other higher levels.

Consider a model with group-specific regression of logit of the success probability $\operatorname{logit}\left(\pi_{i j}\right)$ on a single level-one explanatory variable $\mathrm{X}$.

$$
\operatorname{logit}\left(\pi_{i j}\right)=\log \left[\frac{\pi_{i j}}{1-\pi_{i j}}\right]=\beta_{0 j}+\sum_{h=1}^{k} \beta_{h i j}+U_{0 j}+\sum_{h=1}^{k} U_{h j} X_{h i j}
$$

The term $\sum_{h=1}^{k} U_{h j} X_{h i j}$ can be regarded as a random interaction between group and the explanatory variables. The random intercept variance, $\operatorname{Var}\left(U_{0 j}\right)=\sigma_{0}^{2}$, the random slope variance, $\operatorname{Var}\left(U_{1 j}\right)=\sigma_{1}^{2}$ and the covariance between the random effects, $\operatorname{Cov}\left(U_{0 j}\right.$; $\left.U_{1 j}\right)=\sigma_{01}^{2}$ are called variance components [41].

\section{Likelihood function}

The key ingredients to a Bayesian analysis are the likelihood function, which reflects information about the parameters contained in the data, and the prior distribution, which quantifies what, is known about the parameters before observing data

$$
\begin{aligned}
& Y_{i j} / \pi_{i j} \propto \operatorname{Bernoulli}\left(\pi_{i j}\right) \\
& \operatorname{logit}\left(\pi_{i j}\right)=\log \\
& {\left[\frac{\pi_{i j}}{1-\pi_{i j}}\right]=\beta_{0 j}+\sum_{h=1}^{k} \beta_{h j} X_{h i j}+U_{0 j}+\sum_{h=1}^{k} U_{h j} X_{h i j}}
\end{aligned}
$$

\section{Prior distribution}

The prior distribution is a probability distribution that represents the prior information associated with the parameters of interest. It is a key aspect of a Bayesian analysis. Even if there is prior knowledge about what we are examining, in some cases we might prefer not to use this and let the data speak for themselves. In this case, we wish to express our prior ignorance into the Bayesian system. This leads to non-informative priors. 


\section{The posterior distribution}

All Bayesian inferential conclusions are based on the posterior distribution of the model generated. Using the prior and likelihood function above the full conditional distribution of posterior parameter $\beta_{0}, \beta_{1}, \ldots, \beta_{k}$ is given by:

$$
p\left(\beta_{h} \mid \Omega_{u}, U_{o j}, y_{i j}\right) \propto \prod_{i j} \pi_{i j}^{y_{i j}}\left(1-\pi_{i j}\right)^{1-y_{i j}}
$$

\section{Estimation techniques}

Markov Chain Monte Carlo (MCMC) Methods: The use of Markov chain Monte Carlo (MCMC) methods to evaluate integral quantities has exploded over the last 15 years. To solve a variety of "unsolvable" problems in Bayesian inference we used the Markov Chain Monte Carlo approach [31].Whenever the frequentist results were clear cut (due to a large sample size or a strong association), performing the MCMC method helped to increase the accuracy of the results by narrowing the credible interval, but did not change the direction of hypothesis acceptance. The initial definition required is that of a more primitive concept that underlies for the second MC which is called Markov chains [9]. Although MCMC methods can be used for both frequentist and Bayesian inference, it is more common and easier to use them for Bayesian modeling and MCMC estimation has been restricted to a subset of the potential models that can be fitted in MLwiN [9].

Metropolis-Hastings algorithm: Metropolis-Hastings algorithm is a Markov chain Monte Carlo (MCMC) method for obtaining a sequence of random samples from a probability distribution. The Metropolis-Hastings algorithm works by generating a sequence of sample values. In this thesis the posterior doesn't look like any distribution we know (no Conjugate) and some (or all) of the full conditionals do not look like any distributions we know (no Gibbs sampling for those whose full conditionals we don't know. This is why we were interested to use Metropolis-Hastings algorithm.

\section{Model selection and comparison}

Model selection is to select the best model among several choices based on an evaluation of the performance of the models. A widely used statistic for comparing models in a Bayesian framework is the Deviance Information Criterion. In Bayesian, the lowest expected deviance has the highest posterior probability. Assessing goodness of fit involves investigating how close the values are predicted by the model with that of observed values [6].

\section{Model diagnostic}

The most common ways of checking goodness of fit are: diagnosis for convergence and mixing and posterior-predictive check. We have used the following in our study for convergence tests for the variables are Time Series Plots, kernel density plot, Monte Carlo Standard Error (MCSE), the effective of sample size (ESS) and Partial Autocorrelation Function (PACF). 


\section{Results and discussions}

The response variable considered in this study was the maternal mortality (Death are related to pregnancy or otherwise).The highest percentage of maternal mortality was observed in Afar (2.93\%) followed by Somalia (2.72\%) while the lowest percentage of maternal death was recorded in Addis Ababa (0.46\%) and followed by Dire Dawa $(0.67 \%)$ in 2016. No antenatal visit and rural resident women were the highest frequency of maternal mortality [14]. As study conducted in Ghana on distribution of causes of maternal mortality among different socio-demographic groups Maternal Mortality was generally high for women who lived in rural areas as opposed to urban residence (Placeholder3). No educational attainment and poor wealth index women were also more affected women. Regarding with the age of mother's maternal mortality rate are $2.11 \%, 2.03 \%, 1.71 \%, 1.24 \%, 1.18 \%, 1.13 \%, 0.76 \%$ for mothers whose age are 40-44, 35-39,30-34 25-29, 15-45-49 and 20-24 respectively. This show as 35-39 was the highest percentage of maternal mortality [27]. Based on place of delivery the percentage of maternal mortality those who deliver at home was $2.01 \%$ and $0.59 \%$ those who deliver at health facilities this indicate that most women was delivered at their home and which was developed with the study developed on maternal mortality on Nepal [44]. Likewise, according to the number of antenatal visits the percent of maternal mortality was $2.04 \%, 1.70 \%, 1.12 \%$ and $0.79 \%$ for those, $1-2$ visit, no visit at all, 3-4 visit and more than 4 number of visit respectively. The most recent studies about maternal mortality in Nigeria shows antenatal care visit is significantly related to maternal mortality [37]. The proportion of maternal mortality was differing by place of residence. The highest percent of maternal death has occurred in the rural part of the country (1.69\%) which was high with relative to the urban $(0.85 \%)$ (Table 1).

Pearson chi-square test was applied to know predictors having a strong association with the response variable. The bivariate association between maternal mortality and predictors indicates that mother's status related to pregnancy was strongly associated with place of delivery, age of mother, region, number of antenatal visit, place of residence, wealth index, marital status, contraception, educational attainment, source drinking water [11].and number of child are found significant at 5\% level of significance indicating that, association with maternal mortality.

Test of Heterogeneity: Hence $x^{2}$ test statistic was applied to assess the heterogeneity in the proportion of maternal mortality between regions in Ethiopia. The result obtained by cross tabulation in (Appendix, Table 5) was $x^{2}=38.702, \mathrm{DF}=10$, $p=0.000 \alpha=0.05$, hence we have enough evidence to reject the null hypothesis and conclude that there is heterogeneity of maternal mortality among regions of Ethiopia.

\section{Bayesian multilevel logistic regression analysis of the empty model}

The simplest important specification of the hierarchical linear model is a model in which only the intercept varies between level two units and no explanatory variables are entered in the model. The empty model contains no explanatory variables and it can be considered as a parametric version of assessing heterogeneity of maternal mortality among regions. The variance of the random factor is significant which 
Table 1 the description of the socioeconomic, demographic and environmental factor of maternal mortality in the regional state of Ethiopia

\begin{tabular}{|c|c|c|c|}
\hline Variable & Variable category & No of Mothers & No of death \\
\hline \multirow[t]{2}{*}{ Place of delivery } & Home & 6021 & $121(2.01 \%)$ \\
\hline & Health facilities & 4082 & $24(0.59 \%)$ \\
\hline \multirow[t]{4}{*}{ No of antenatal } & No antenatal & 3467 & $59(1.70 \%)$ \\
\hline & 1-2 antenatal & 2007 & $41(2.04 \%)$ \\
\hline & 3-4 antenatal & 2597 & $29(1.12 \%)$ \\
\hline & $\geq 5$ antenatal & 2032 & $16(0.78 \%)$ \\
\hline \multirow[t]{2}{*}{ Place of residence } & Urban & 3044 & $26(0.85 \%)$ \\
\hline & Rural & 7059 & 119 (1.69\%) \\
\hline \multirow[t]{3}{*}{ Educational attainment } & No education & 5922 & $111(1.87 \%)$ \\
\hline & Primary & 2770 & $26(0.94 \%)$ \\
\hline & Secondary and above & 1411 & $8(0.57 \%)$ \\
\hline \multirow[t]{3}{*}{ Wealth index } & Poor & 4753 & $96(2.02 \%)$ \\
\hline & Medium & 1822 & $29(1.59 \%)$ \\
\hline & Rich & 3528 & $20(0.57 \%)$ \\
\hline \multirow[t]{2}{*}{ Contraceptive } & Not using & 7557 & 119 (1.57\%) \\
\hline & Use & 2546 & $26(1.02 \%)$ \\
\hline \multirow[t]{4}{*}{ Marital status } & Not married & 698 & $19(2.72 \%)$ \\
\hline & Married & 8411 & $11(1.32 \%)$ \\
\hline & separated & 280 & $2(0.71 \%)$ \\
\hline & Widow/divorced & 714 & $13(1.82 \%)$ \\
\hline \multirow[t]{4}{*}{ No of children } & No child & 696 & $4(0.57 \%)$ \\
\hline & 1-2 child & 3091 & $31(1.00 \%)$ \\
\hline & 3-4 child & 3383 & $60(1.77 \%)$ \\
\hline & $\geq 5$ child & 2933 & $50(1.71 \%)$ \\
\hline \multirow[t]{3}{*}{ Source of drinking water } & Piped water & 3787 & $32(0.84 \%)$ \\
\hline & Tube well water & 2576 & $39(1.98 \%)$ \\
\hline & Surface water & 3740 & $74(1.51 \%)$ \\
\hline
\end{tabular}

Table 2 Bayesian multilevel logistic regression of empty model

\begin{tabular}{lclll}
\hline Model & Coefficient & SD & MCSE & 95\%Cr.I \\
\hline Fixed intercept $\left(\beta_{0 j}\right)$ & -5.257 & 0.359 & 0.0046 & $(-6.048,-4.545)$ \\
Random intercept $\operatorname{var}\left(u_{0 j}\right)=\sigma_{u 0}^{2}$ & 3.966 & 1.811 & 0.0252 & $(0.997,8.012)$ \\
\hline
\end{tabular}

indicates that there are regional differences in maternal mortality. The regional variation of maternal mortality is 3.966 (1.811) which is significant (Table 2).

The overall mean of maternal mortality is estimated that $\beta_{0}=-5.257$ found to be significant, suggest that evidence of regional effects on maternal mortality. The variance of the random factor is significant which indicates that there are regional differences in maternal mortality and thus, Bayesian multilevel analysis can be considered as an appropriate approach for further analysis. 


\section{Bayesian multilevel logistic regression random intercept model}

The random intercept model is where the intercept is allowed to vary across regions after controlling for covariates of pregnancy-related mortality. The results from the random intercept model showed that the random intercept $\beta_{0 j}$ is significant implying that the average pregnancy-related death is differing from region to region. The results from the random intercept model showed that the random intercept $\beta_{0 j}$ is significant implying that the average pregnancy-related death is differing from region to region. The result shows that the variance of the random effect is significant which indicates that there are regional differences in maternal mortality in the given data set.

\section{Bayesian multilevel logistic regression random coefficient model}

The odds of maternal death in health facilities was $58 \%(\mathrm{OR}=0.42)$ times less likely than the odds of maternal death in a home. Regarding too number of antenatal visit the odds of maternal mortality for those who 3-4 visited and more than 5 number of antenatal visit was 0.81 and 0.64 less likely than the odds of no antenatal visit by assuming other variable constant respectively. The study indicated that the likelihood of mothers experiencing maternal mortality reduces with the number of visits [50].

Another finding of this study indicates that the age of individual women is significantly associated with maternal mortality with $95 \%$ credible interval [16]. Particularly, the odds of maternal mortality with age of mothers between 20 and 24 years was 1.17 times more likely to be dead than the odds of maternal mortality aged between 15 and 19 years and the odds of maternal death aged between 25 and 29 years were 1.98 times more likely to be dead than the odds of mothers age between 15 and 19 years. Educational attainment has a significant contribution to maternal mortality [32]. The odds of pregnancyrelated death of women for primary education was about $45 \%(\mathrm{OR}=0.55)$ less likely than the odds of pregnancy-related death of women who have no education (illiterate) and the odds of maternal mortality for secondary and above education were about $66 \%$ $(\mathrm{OR}=0.34)$ less likely than the odds of maternal mortality for who has no education by assuming another factor constant [7]. Additional Women education has a positive and significant effect on the place of delivery at the time of childbirth [3]

The Bayesian multilevel logistic regression analysis result displayed in Table 3 below, also estimates the variance of random effect at the regional level, $\operatorname{var}\left(U_{0 j}\right)$. Thus, the value of $\operatorname{var}\left(u_{0 j}\right)=4.085$ indicate there was significant variation (which means the $95 \%$ credible intervals is greater than zero). This confirmed the significance of the regional difference in maternal mortality in the regional state of Ethiopia. The researcher tried to identify to see the level of variation; that the intra-region correlation coefficient ICC is estimated as $\rho \rho=\frac{4.085}{4.085+3.29}=0.5538$. This means that about $55.38 \%$ of the total variability in maternal mortality is due to differences across regions, with the remaining unexplained $44.62 \%$ attributable to individual differences.

This model contains a random slope for wealth index and the number of living children; which means that it allows the effect of the coefficient of this variable to vary from region to region. This model is more appropriate than the previous model for the variables being used since from wealth index category rich has fixed coefficient $-1.296(0.308)$, which suggests that this is the strong predictor and from wealth index 
Table 3 Bayesian Estimates for Random coefficient model

\begin{tabular}{|c|c|c|c|c|c|}
\hline Fixed effect & Categories & Estimates & SD & MC error & $95 \% \mathrm{Cl}$ \\
\hline Intercept & & -4.955 & 0.880 & 0.0979 & $(-6.757,-3.407)$ \\
\hline \multirow[t]{2}{*}{ P.delivery } & Home(ref) & & & & \\
\hline & H.facilities & -0.864 & 0.286 & 0.001 & $(-1.423,-0.306)$ \\
\hline \multirow[t]{4}{*}{ №Ante.visit } & No visit(ref) & & & & \\
\hline & $1-2$ visit & 0.352 & 0.253 & 0.0009 & $(-0.148,0.851)$ \\
\hline & $3-4$ visit & -0.210 & 0.273 & 0.0009 & $(-0.759,0.311)$ \\
\hline & $5+$ visit & -0.46 & 0.344 & 0.0012 & $(-1.142,0.192)$ \\
\hline \multirow[t]{4}{*}{ № children } & No child(ref) & & & & \\
\hline & $1-2$ child & 1.219 & 0.717 & 0.0075 & $(-0.106,2.654)$ \\
\hline & 3-4 child & 1.925 & 0.738 & 0.0091 & $(0.537,3.426)$ \\
\hline & $5+$ child & 1.628 & 0.749 & 0.0416 & $(0.174,3.060)$ \\
\hline \multirow[t]{2}{*}{$P$ residence } & Urban(ref) & & & & \\
\hline & Rural & 0.666 & 0.447 & 0.0037 & $(-0.192,1.598)$ \\
\hline \multirow[t]{3}{*}{ E attainment } & No educ (ref) & & & & \\
\hline & Primary educ & -0.589 & 0.267 & 0.0009 & $(-1.115,-0.076)$ \\
\hline & Sec.and above & -1.069 & 0.465 & 0.0016 & $(-2.040,-0.167)$ \\
\hline \multirow[t]{3}{*}{ Wealth index } & Poor(ref) & & & & \\
\hline & Middle & -0.342 & 0.270 & 0.001 & $(-0.894,0.189)$ \\
\hline & Rich & -1.296 & 0.308 & 0.0011 & $(-1.927,-0.721)$ \\
\hline \multirow[t]{2}{*}{ Contraceptive } & Not use(ref) & & & & \\
\hline & Use & -0.049 & 0.274 & 0.0038 & $(-0.581,0.469)$ \\
\hline \multirow[t]{3}{*}{ Random effect } & $\sigma_{u 0}^{2}$ & 4.085 & 1.222 & 0.051 & $(1.659,3.885)$ \\
\hline & $\sigma_{u 20}^{2}$ & 31.193 & 17.983 & 1.074 & $(6.551,70.06)$ \\
\hline & $\sigma_{13}^{2}$ & 4.168 & 3.073 & 0.138 & $(0.644,12.69)$ \\
\hline
\end{tabular}

category rich women were significantly less likely than poor women. It is necessary to see that the effect of wealth index on maternal mortality varies from region to region in Ethiopia which implies that there is a considerable variation in the effects of wealth index and the number of living children [19]. The region wise intercept $\left(u_{0 j}\right)$ and slope (wealth index $=u_{0 j}$ ) vary significantly, that is, there is a significant variation in the effects of these explanatory variables across the regions. Another concept under this study the researcher revealed that the variance of the random slopes. The values of $\operatorname{var}\left(\mathrm{u}_{0 j 20}\right) u_{0 j, 20}=31.193$ with credible interval of $(95 \%$ CI $6.551,70.06)$ and $\sigma_{13}^{2}=4.168$ with $(95 \%$ CI $0.644,12.69)$ the interval was greater than zero. This indicates that the random slope of wealth index and the number of living children in the region is significant. This means that the wealth index and the number of living children factor for maternal mortality vary from region to region.

\section{Model comparison}

Bayesian deviance information criterion showed that Bayesian multilevel random coefficient model is the most significant model and best fit the data. The diagnostic $\mathrm{DIC}=$ Deviance $+2 * \mathrm{pD}$ where $\mathrm{pD}$ is the 'effective number of parameters' which can be calculated from the chain as the difference between the mean deviance (Dbar) in the chain and the deviance at the mean values for the parameters (D (bar)). 
Table 4 DIC values for model comparison

\begin{tabular}{lcccc}
\hline \multicolumn{4}{l}{ Bayesian Deviance Information Criterion (DIC) for model comparison } & \\
\hline Model & $\widehat{D}$ & $\mathbf{D}(\widehat{\boldsymbol{\theta}})$ & Pd & DIC \\
\hline Null model & 1222.06 & 1061.04 & 161.02 & 1383.09 \\
Random intercept & 1110.73 & 939.70 & 171.03 & 1281.76 \\
Random coefficient & 1047.24 & 862.73 & 184.51 & 1231.75 \\
\hline
\end{tabular}

From the result of Table 4 below, the DIC diagnostics of random intercept Bayesian multilevel logistic regression model is reduced by 101.33 from the Bayesian multilevel logistic regression of an empty model. This show as adding covariate variables to the model indicates how the variable is determined maternal mortality. Thus; Bayesian multilevel logistic regression for random intercept was the better model as compared to Bayesian multilevel for an empty model. The DIC diagnostics of Bayesian multilevel logistic regression of random coefficient model is reduced by 50.01 from Bayesian multilevel for random intercept so, this Bayesian multilevel random coefficient model is a great improvement suggesting that this model is the appropriate model than a Bayesian multilevel empty model and Bayesian multilevel for intercept model to determine the maternal mortality factors.

Therefore, this Bayesian deviance information criterion showed that Bayesian multilevel random coefficient model is the most significant model and best fit the data. The average deviance from the complete set of iterations $(\widehat{D})$ also decreased from an empty model to random intercept and from random intercept to the random coefficient model. $\mathrm{D}(\widehat{\theta})$ shows that the deviance at the expected value of the unknown parameters and it also shows the decreasing trend from an empty model to random intercept and from random intercept to the random coefficient model.where $(\widehat{D})$ : The average deviance from the complete set of iterations, $\mathrm{D}(\widehat{\theta})$ : The deviance at the expected value of the unknown parameters, pD: The Estimated degrees of freedom consumed in the fit, ieDbar- D(theta Bar) and DIC: Fit + Complexity; Dbar + PD.

\section{Assessment of model convergence}

The plots of all statistically significant covariates indicated that none of the coefficients have bimodal density and hence the simulated parameter values have converged. The ACF measure show correlated the values in the chain are with their close neighbors. The lag is the distance between the two chains to be compared. So, the plots displayed in Fig. 2 below indicate low autocorrelation and efficient sampling as we have seen it. The Partial Autocorrelation Function (PACF) measures discrepancies from such a process and so should normally have values 0 after lag 1 which shows again convergence. The Monte Carlo Standard Error (MCSE) is an indication of how much error is in the estimate due to the fact that Markov chain Monte Carlo (MCMC) is used. As the number of iteration increased the Monte Carlo Standard Error (MCSE) was decreased as we have seen from the graph. 

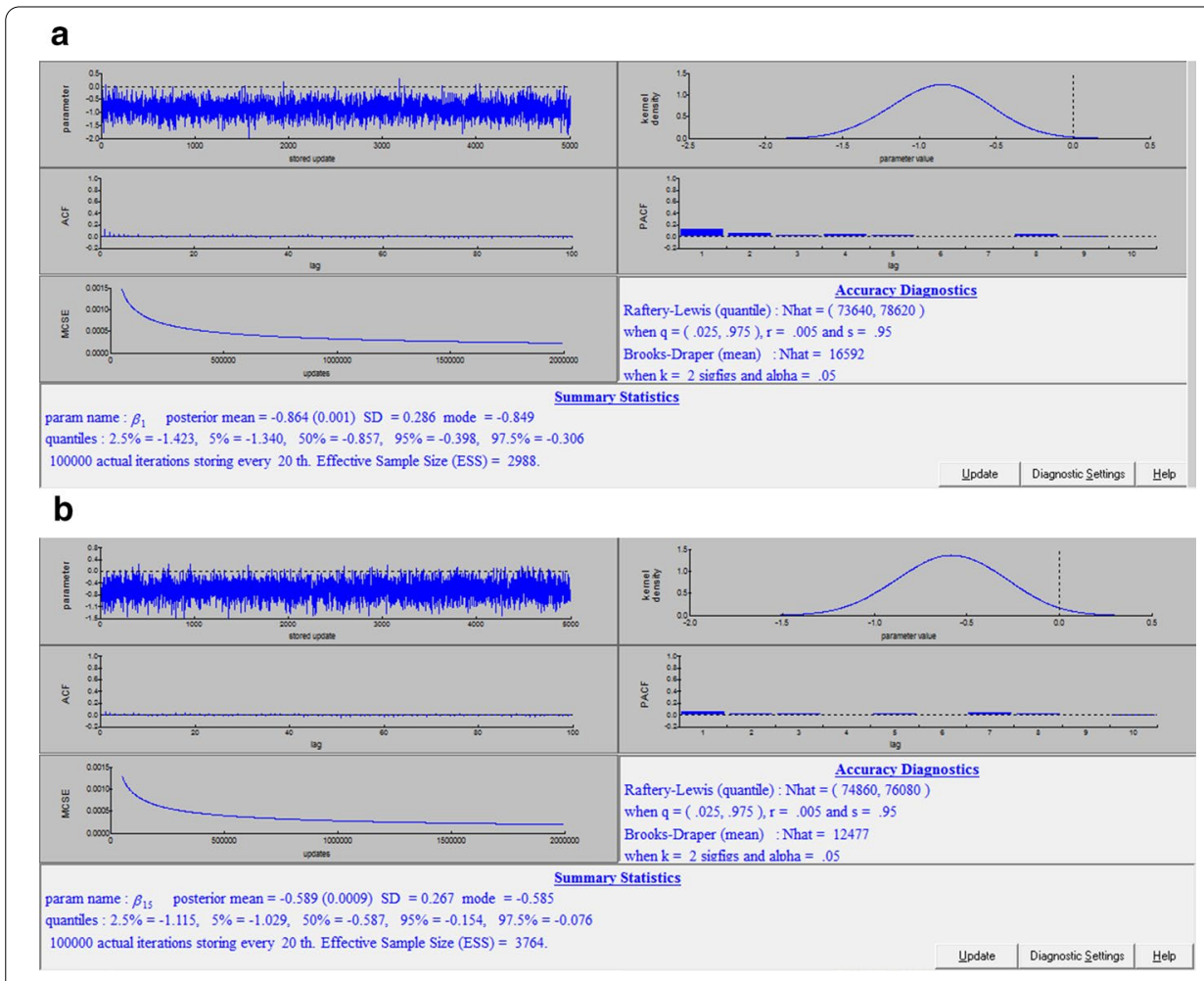

Fig. 2 a convergences for $\beta_{1}$.b convergences for $\beta_{15}$

\section{Discussion}

These studies were attempted to identify some socio-economic and demographic determinants of maternal mortality in Ethiopia using 2016 EDHS data. Accordingly, the descriptive method and Bayesian Multilevel logistic regression were used in the analyses. The variables, having the significant association with maternal mortality (based on Chi-square test of association) place of delivery, Antenatal care, Mother's age at birth, Place of residence, Region, mothers education, Marital status [36]. Wealth index, Contraceptive [45], number of living children, a source of drinking water [42]. The Bayesian multilevel logistic regression empty model, the Bayesian multilevel logistic regression random intercept model, and Bayesian multilevel logistic regression random coefficient model were used in this study.

Based on results of descriptive statistics the probability of maternal mortality for Afar and Somali was high as compared to the other region and also the number of maternal mortality for urban women is less likely than that of rural, place of delivery in health facilities was less than that of home [26]. Coming to the inference parts the analysis of the final model indicated that one of the significant factors of maternal mortality in this study was the place of delivery. This show as the women who delivered at home has died more than those who deliver at health facilities. Access to proper medical attention and hygienic conditions during delivery can reduce the risk of complications and infections that may lead to death or serious illness for the mother and/or baby [30].

Another finding was showed that wealth index was an important determinant of maternal mortality. The maternal mortality from the low-income family has more likely 
died than that of from high income or rich family the result for household wealth index being significant is also consistent with the previous study by [4].

The number of living children were one of the other significantly associated with pregnancy-related death. Which mean that as the number of children increases the chance of pregnancy-related death also high. Another message we have understood from these study was the level of education of women also significantly associated with pregnancyrelated death. The study showed that educational attainment was an important determinant for pregnancy-related death and had negative effects [21]. Another objective of the study was to identify the regional variation and our studies suggest that the Bayesian multilevel coefficient model indicated that there were regional variations of maternal mortality. From the result of the model adequacy Bayesian multilevel logistic random coefficient model is the best-fitted model [43].

The analysis based on Bayesian multilevel logistic regression provided estimates for variances of the random effects and interclass correlations. The estimates for each level were different, suggesting that the variance components of maternal mortality were different at individual and regional levels. This means that the sources of variations are individuals and regions. The result of Bayesian multilevel logistic regression model comparison indicates that the random coefficient Bayesian multilevel logistic regression model best fits the model than the null model and random intercept model of the Bayesian multilevel logistic regression model. Therefore, the researcher suggests that Bayesian multilevel logistic regression for random coefficient were the best fit of the data and the interpretation was depending on random coefficients.

\section{Conclusions}

The study data was taken from the Ethiopian Demographic Health Survey conducted by the Central Statistical Agency (CSA) of Ethiopia in 2016. An inference is a fully Bayesian multilevel model based on recent Markov chain Monte Carlo techniques. The study was identified socio-economic, demographic and environmental proximate variables as determinants of maternal mortality in the country and the gap from classical by checking the level of variation within and between regions. From the methodological aspect, it was found that Bayesian multilevel random coefficient model is better compared to empty (null) model and random intercept model in fitting the data and in explaining the variations of pregnancy-related mortality across regional levels of Ethiopia. In addition, from the empty model and random intercept model, the overall variance of the constant term was found to be statistically significant, implying the existence of a difference in pregnancy-related mortality in Ethiopia. The regional variations were high for the Bayesian multilevel empty model than the Bayesian multilevel for random intercept and lower for Bayesian multilevel for a random coefficient model to explaining the regional variation of 2016.

\section{Recommendation}

The findings of this study have some important policy implications and the identification of factors those are significantly associated with a maternal mortality. Depending on the above important findings, the researcher suggests the following recommendations for researchers and policymakers: 
- Although the variation across the regions has been addressed with this study, the distribution for the prevalence of maternal mortality and the issue of identifying the hot-spot-area is not covered here. Therefore, the researchers are recommended to extend this study with the application of spatial models.

- The data of this study was basically secondary which have the problem of missing data and the expected potential variables were also not availed. Thus, researchers should have to conduct the study on separate regions with the same models of this study.

- This study was limited to identifying the socio-demographic factors. However, there are other major causes of maternal mortality. Hence, we recommended researchers so that to study the significance of those causes by considering only maternal mortality using the Poisson model and its extension.

- The government and other concerned bodies should have to take attention to control the significant factors that lead to maternal mortality like mothers' educational level have to upgrade, Mothers have to be encouraged to reduce home delivery and attitude toward having the small number of children has to be raised.

\section{Acknowledgements}

First and foremost, glory to Almighty God for giving full of health, peace, the opportunity to pursue our University Wollega and Mettu University for offering our opportunity and financial support during studies.

We would like gratefully and sincerely to thank, Akalu Banbeta (Ph.D. scholar), for his invaluable comments, suggestions, and patience during the entire time of the study. data.

We sincere thanks are due to the Central Statistical Agency of Ethiopia for providing the Demographic Health Survey

\section{Authors' contributions}

SJ made contributions on conceptualized the research problem, designed the study, performed statistical analysis, interpretation of data and revised and drafting the manuscript. DJ was involved in, revision of the research design, data analysis and revision of the manuscript for publication. Both authors read and approved the final manuscript.

\section{Funding}

None.

Availability of data and materials

The data set was taken from the Ethiopian Demographic and Health Survey (EDHS 2016) website.

Competing interests

The authors declare that not competing interest were available.

Author details

1 Department of Statistics, College of Natural and Computational Sciences, Wollega University, Nekemte, Ethiopia.

${ }^{2}$ Department of Statistics, College of Natural and Computational Science, Mettu University, Mettu, Ethiopia.

\section{Appendix}

See Table 5. 
Table 5 Test of heterogeneity

\begin{tabular}{lllll}
\hline Variables & Total & DF & Chi-sqr & p-value \\
\hline Region & 10,103 & 10 & 38.70 & 0.000 \\
Age of mother & 10,103 & 6 & 15.89 & 0.014 \\
Place of delivery & 10,103 & 1 & 34.76 & 0.000 \\
No of antenatal & 10,103 & 3 & 14.87 & 0.002 \\
Place residence & 10,103 & 1 & 10.40 & 0.001 \\
Educational attainment & 10,103 & 2 & 20.42 & 0.000 \\
Wealth index & 10,103 & 2 & 30.60 & 0.000 \\
Marital status & 10,103 & 3 & 10.74 & 0.020 \\
No of children & 10,103 & 3 & 11.97 & 0.007 \\
Contraceptive & 10,103 & 1 & 4.12 & 0.042 \\
Source of drinking water & 10,103 & 2 & 17.25 & 0.000 \\
\hline
\end{tabular}

Received: 12 September 2020 Accepted: 9 December 2020

Published online: 17 February 2021

\section{References}

1. Abdulai S. Analysis of maternal mortality in Wa District (Doctoral dissertation); 2015.

2. Acquah HD. Bayesian logistic regression modelling via Markov Chain Monte Carlo Algorithm. J Soc Dev Sci. 2013;4(4):193-7.

3. Asamoah BO. Distribution of causes of maternal mortality among different socio-demographic groups in Ghana; a descriptive study. BMC Public Health. 2011;11(1):159.

4. Bayati MV. Determinants of maternal mortality in Eastern Mediterranean region: a panel data analysis. Med J Islamic Republic of Iran. 2016;30:360.

5. Berhan Y. Review of maternal mortality in Ethiopia: a story of the past 30 years. Ethiop J Health Sci. 2014:24:3-14

6. Bewick V, Cheek L, Ball J. Logistic regression. Crit Care. 2005;9:112-8. https://doi.org/10.1186/cc3045.

7. Bicego GB. The effect of AIDS on maternal mortality in Malawi and Zimbabwe. Aids. 2002;16(7):1078-81.

8. Browne WJ. A comparison of Bayesian and likelihood-based methods for fitting multilevel models. Bayesian Anal. 2006:1(3):473-514.

9. Browne WJ. MCMC estimation in MLwiN. ISBN: 0-9544036-4-9 Centre of Multilevel Modelling Institute of Education. University of London; 2003.

10. Collet D. Modeling binary data. London: Chapman and Hall; 1991.

11. Cheng JJ-W. An ecological quantification of the relationships between water, sanitation and infant, child, and maternal mortality. Environ Health. 2012;11(1):4.

12. CheruKitabo A, Damtie ET. Bayesian multilevel analysis of utilization of antenatal care services in Ethiopia. Hindawi Comput Math Methods Med. 2020. https://doi.org/10.1155/2020/8749753.

13. CSA. Ethiopia Demographic and Health Survey Data. Addis Ababa: Central Statistical Authority (Ethiopia) and ORC Macro; 2016.

14. Dahiru T, Oche OM. Determinants of antenatal care, institutional delivery and postnatal care services utilization in Nigeria. Pan Afr Med J. 2015;21(1):321.

15. Gelman A, Hill J. Data analysis using regression and multilevel/hierarchical models. Cambridge: Cambridge University Press; 2006.

16. Geubbels EA. Health \& demographic surveillance system profile: the Ifakara rural and urban health and demographic surveillance system (Ifakara HDSS). Int J Epidemiol. 2015;44(3):848-61.

17. Grzenda W. Factors related to unemployment, the advantages of Bayesian methods over classical methods in the context of credible intervals. InfSystManag. 2015;4(1):53-63.

18. Hastings W. Monte Carlo sampling methods using Markov chains and their applications. In: Biometrika, vol. 57; 1970. p. $97-109$

19. Hedegaard KH. Factors associated with maternal mortality in rural Guinea-Bissau. A longitudinal population-based study. BJOG Int J ObstetGynaecol. 2002;109(7):792-9.

20. Hill KT. Estimates of maternal mortality worldwide between 1990 and 2005: an assessment of available data. The Lancet. In: Maternal mortality working group; 2007. 370(9595): 1311-1319.

21. Jat TR. Factors affecting the use of maternal health services in Madhya Pradesh state of India: a multilevel analysis. Int J Equity Health. 2011;10(1):59.

22. Jarso S, Haji Y, Gebretsadik A. Determinants of maternal death in a pastoralist area of Borena Zone, Oromia Region, Ethiopia: unmatched case-control study. Obstet Gynecol Int. 2019. https://doi.org/10.1155/2019/5698436.

23. Kullima AA, Kawuwa MB, Audu BM, Geidam AD, Mairiga AG. Trends in maternal mortality in a tertiary institution in Northern Nigeria. Ann Afr Med. 2009;8(4):221-4.

24. Larget B, Simon DL. Markov chain Monte Carlo algorithms for the Bayesian analysis of phylogenetic trees. Mol Biol Evol. 1999;16(6):750-9.

25. Legesse T. Trends and causes of maternal mortality in Jimma University specialized Hospital, southwest Ethiopia: a matched case-control study. Int J Women's Health. 2017;9:307. 
26. Liu ZZ. RH knowledge and service utilization among unmarried rural-to-urban migrants in three major cities, China. BMC Public Health. 2011;11(1):74.

27. Mbaruku GM. Causes and risk factors for maternal mortality in rural Tanzania-Case of Rufiji health and demographic surveillance site (HDSS). Afr J Reprod Health. 2013;17(3):119-30.

28. McAlister CA. Female education and maternal mortal- ity, A worldwide survey. Halifax: Department of Obtetrics and Gynaecology, Dalhouse University; 2006.

29. Mekonnen ZA. Multilevel analysis of individual and community level factors associated with institutional delivery in Ethiopia. BMC Res Notes. 2015;8(1):376.

30. Melaku YA. linkages in maternal health care services - evidence on antenatal care and institutional delivery from a community-based longitudinal study in Tigray region, Ethiopia. BMC Pregnancy Childbirth. 2014;14(1):18.

31. Mira A. MCMC methods to estimate Bayesian parametric models. In: E. BV, Handbook of statistics, vol. 25; 2005.

32. Navaneetham K. Utilization of maternal health care services in Southern India. SocSci Med. 2002;55(10):1849-69.

33. Ntoimo LF. Prevalence and risk factors for maternal mortality in referral hospitals in Nigeria: a multicenter study. Int $J$ Women's Health. 2018;10:69-76.

34. Ogunsakin RE. Bayesian inference on malignant breast cancer in Nigeria: a diagnosis of MCMC convergence. Asian Pac J Cancer Prev APJCP. 2017;18(10):2709.

35. Ojo OB, Lougue $S$, Woldegerima WA. Bayesian generalized linear mixed modeling of Tuberculosis using informative priors. PLoS ONE. 2017;12(3):e0172580.

36. Ojua TD. African cultural practices and health implications for Nigeria rural development. Int Rev Manag Bus Res. 2013;2(1):8.

37. Omo-Aghoja EA. Maternal mortality at the Central Hospital, Benin City Nigeria African. J Reprod Health. 2008;12(3):17-26.

38. Pascale Salameh MW. Bayesian and frequents comparison for epidemiologists: a non mathematical application on logistic regressions. Open Epidemiol J. 2014;7:17-26.

39. Rue HM. Approximate Bayesian inference for latent Gaussian models by using integrated nested Laplace approximations. J R Stat Soc Ser B. 2009;71 (2):319-92.

40. Shah IH. Maternal mortality and maternity care from 1990 to 2005: uneven but important gains. Reprod Health Matters. 2007;15(30):17-27.

41. Snijders TAB, Bosker RJ. Multilevel analysis: an introduction to basic and advanced multilevel modeling. London, Thousand Oaks, New Delhi: SAGE Publications; 1999.

42. Sommer JM. Water, sanitation, and health in sub-Saharan Africa: a cross-national analysis of maternal and neo-natal mortality. Human Ecol Rev. 2015;22(1):129-52.

43. Spiegelhalter DJ, Best NG, Carlin BP, Van Der Linde A. Bayesian measures of model complexity and fit. J R Statist Soc B. 2002;64(4):583-639.

44. Suwal JV. Maternal mortality in Nepal: Unraveling the complexity. Can Stud Popul. 2008;35(1):1-26.

45. Stover JA. How increased contraceptive use has reduced maternal mortality. Maternal Child Health J. 2010;14(5):687-95.

46. UNICEF. World Health Organization. Trends in maternal mortality: 1990-2015: estimates from World Bank Group and the United Nations Population Division. World Health Organization. New York: UNFPA, WHO.

47. Weyesa JB. Prevalence and risk factors associated with maternal mortality in Mizan-Aman Hospital, Bench Maji Southwest Ethiopia. J Women's Health Care. 2015;4(274):2167.

48. WHO. Maternal mortality database: tables. Geneva: WHO; 2007.

49. WHO, UNICEF. Trends in maternal mortality: 1990-2015: estimates from. New York: WHO UNICEF, UNFPA, World Bank Group and the United Nations Population Division; 2015.

50. Yaya YD. Maternal mortality in rural south Ethiopia: outcomes of community-based birth registration by health extension workers. PLoS ONE. 2015;10(3):e0119321.

51. Yego FD. Risk factors for maternal mortality in a Tertiary Hospital in Kenya: a case control study. BMC Pregnancy Childbirth. 2014;14(1):38.

\section{Publisher's Note}

Springer Nature remains neutral with regard to jurisdictional claims in published maps and institutional affiliations.

\section{Submit your manuscript to a SpringerOpen ${ }^{\circ}$ journal and benefit from:}

- Convenient online submission

- Rigorous peer review

- Open access: articles freely available online

- High visibility within the field

- Retaining the copyright to your article

Submit your next manuscript at $\boldsymbol{\nabla}$ springeropen.com 